\title{
The Third Data Release of the Sloan Digital Sky Survey
}

\section{Citation}

Abazajian, Kevork, Jennifer K. Adelman-McCarthy, Marcel A. Agüeros, Sahar S. Allam, Kurt S. J. Anderson, Scott F. Anderson, James Annis, et al. 2005. "The Third Data Release of the Sloan Digital Sky Survey." The Astronomical Journal 129 (3) (March): 1755-1759. doi:10.1086/427544.

\section{Published Version}

doi:10.1086/427544

\section{Permanent link}

http://nrs.harvard.edu/urn-3:HUL.InstRepos:33370051

\section{Terms of Use}

This article was downloaded from Harvard University's DASH repository, and is made available under the terms and conditions applicable to Other Posted Material, as set forth at http:// nrs.harvard.edu/urn-3:HUL.InstRepos:dash.current.terms-of-use\#LAA

\section{Share Your Story}

The Harvard community has made this article openly available.

Please share how this access benefits you. Submit a story.

Accessibility 


\section{THE THIRD DATA RELEASE OF THE SLOAN DIGITAL SKY SURVEY}

Kevork Abazajian, ${ }^{1}$ Jennifer K. Adelman-McCarthy, ${ }^{2}$ Marcel A. Agüeros, ${ }^{3}$ Sahar S. Allam, ${ }^{2}$ Kurt S. J. Anderson, ${ }^{4,5}$ Scott F. Anderson, ${ }^{3}$ James Annis, ${ }^{2}$ Neta A. Bahcall, ${ }^{6}$ Ivan K. Baldry, ${ }^{7}$ Steven Bastian, ${ }^{2}$ Andreas Berlind, ${ }^{8,9,10}$ Mariangela Bernardi, ${ }^{1,12}$ Michael R. Blanton, ${ }^{10}$ John J. Bochanski, JR., ${ }^{3}$ William N. Boroski, ${ }^{2}$ Howard J. Brewington, ${ }^{4}$ John W. Briggs, ${ }^{13}$ J. Brinkmann, ${ }^{4}$ Robert J. Brunner, ${ }^{14}$ Tamás Budavári, ${ }^{7}$ Larry N. Carey, ${ }^{3}$ Francisco J. Castander, ${ }^{15}$ A. J. Connolly, ${ }^{11}$ Kevin R. Covey, ${ }^{3}$ István Csabai, ${ }^{7,16}$ Julianne J. Dalcanton, ${ }^{3}$ Mamoru Doi, ${ }^{17}$ Feng Dong, ${ }^{6}$ Daniel J. Eisenstein, ${ }^{18}$ Michael L. Evans, ${ }^{3}$ Xiaohui Fan, ${ }^{18}$ Douglas P. Finkbeiner, ${ }^{6,19}$ Scott D. Friedman, ${ }^{20}$ Joshua A. Frieman, ${ }^{2,8,9}$ Masataka Fukugita, ${ }^{21}$ Bruce Gillespie, ${ }^{4}$ Karl Glazebrook, ${ }^{7}$ Jim Gray $^{22}{ }^{2}$ Eva K. Grebel, ${ }^{23}$ James E. Gunn, ${ }^{6}$ Vijay K. Gurbani, ${ }^{2,24}$ Patrick B. Hall ${ }^{6,25}$ Masaru Hamabe, ${ }^{26}$ Daniel Harbeck, ${ }^{27}$ Frederick H. Harris, ${ }_{7}^{28}$ Hugh C. Harris, ${ }^{28}$ Michael Harvanek, ${ }^{4}$ Suzanne L. Hawley, ${ }^{3}$ Jefrrey Hayes, $^{29}$ Timothy M. Heckman, ${ }^{7}$ John S. Hendry, ${ }^{2}$ Gregory S. Hennessy ${ }^{30}$ Robert B. Hindsley, ${ }^{31}$ Craig J. Hogan, ${ }^{3}$ David W. Hogg, ${ }^{10}$ Donald J. Holmgren, ${ }^{2}$ Jon A. Holtzman, Shin-Ichi Ichikawa, ${ }^{32}$ Takashi Ichikawa, ${ }^{33}$ Željko Ivezić, ${ }^{6}$ Sebastian Jester, ${ }^{2}$ David E. Johnston, ${ }^{6}$ Anders M. Jorgensen, ${ }^{34}$ Mario Jurić, ${ }^{6}$ Stephen M. Kent, ${ }^{2}$ S. J. Kleinman, ${ }^{4}$ G. R. Knapp, ${ }^{6}$ Alexei Yu. Kniazev, ${ }^{23}$ Richard G. Kron, ${ }^{2,8}$ Jurek Krzesinski, ${ }^{4,35}$ Donald Q. Lamb, ${ }^{8,36}$ Hubert Lampeitl, ${ }^{2}$ Brian C. Lee, ${ }^{37}$ Huan Lin, ${ }^{2}$ Daniel C. Long, ${ }^{4}$ Jon Loveday, ${ }^{38}$ Robert H. Lupton, ${ }^{6}$ Ed Mannery, ${ }^{3}$ Bruce Margon, ${ }^{20}$ David Martínez-Delgado, ${ }^{23}$ Takahiko Matsubara, ${ }^{39}$ Peregrine M. McGehee, ${ }^{5,40}$ Timothy A. McKay, ${ }^{41}$ Avery Meiksin, ${ }^{42}$ Brice Ménard, ${ }^{43}$ Jefrerey A. Munn, ${ }^{28}$ Thomas Nash, ${ }^{2}$ Eric H. Neilsen, Jr., ${ }^{2}$ Heidi Jo Newberg, ${ }^{44}$ Peter R. Newman, ${ }^{4}$ Robert C. Nichol, ${ }^{45}$ Tom Nicinski, ${ }^{2,46}$ Maria Nieto-Santisteban, ${ }^{20}$ Atsuko Nitta, ${ }^{4}$ Sadanori Okamura, ${ }^{47}$ William O'Mullane, ${ }^{7}$ Russell Owen, ${ }^{3}$ Nikhil Padmanabhan, ${ }^{48}$ George Pauls, ${ }^{6}$ John Peoples, ${ }^{2}$ Jefrer R. Pier, ${ }^{28}$ Adrian C. Pope, ${ }^{7}$ Dimitri Pourbaix, ${ }^{6,49}$ Thomas R. Quinn, ${ }^{3}$ M. Jordan Raddick, ${ }^{7}$ Gordon T. Richards, ${ }^{6}$ Michael W. Richmond, ${ }^{50}$ Hans-Walter Rix, ${ }^{23}$ Constance M. Rockosi, ${ }^{51}$ David J. Schlegel, ${ }^{6}$ Donald P. Schneider, ${ }^{52}$ Joshua Schroeder, ${ }^{6,53}$ Ryan Scranton, ${ }^{11}$ Maki Sekiguchi, ${ }^{54}$ Erin Sheldon, ${ }^{8,9}$ Kazu Shimasaku, ${ }^{47}$ Nicole M. Silvestri, ${ }^{3}$ J. Allyn Smith, ${ }^{34,55}$ Vernesa Smolčić, ${ }^{56}$ Stephanie A. Snedden, ${ }^{4}$ Albert Stebbins, ${ }^{2}$ Chris Stoughton, ${ }^{2}$ Michael A. Strauss, ${ }^{6}$ Mark SubbaRao, ${ }^{8,57}$ Alexander S. Szalay, ${ }^{7}$ István Szapudi, ${ }^{58}$ Paula Szkody, ${ }^{3}$ Gyula P. Szokoly, ${ }^{59}$ Max Tegmark, ${ }^{60}$ Luis Teodoro, ${ }^{1}$ Aniruddha R. Thakar, ${ }^{7}$ Christy Tremonti, ${ }^{18}$ Douglas L. Tucker, ${ }^{2}$ Alan Uomoto, ${ }^{7,61}$

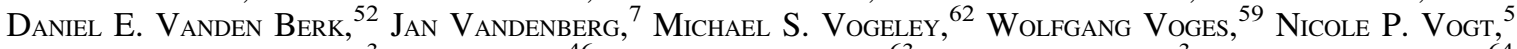

Lucianne M. Walkowicz, ${ }^{3}$ Shu-i Wang, ${ }^{46}$ David H. Weinberg, ${ }^{63}$ Andrew A. West, ${ }^{3}$ Simon D. M. White, ${ }^{64}$ Brian C. Wilhite, ${ }^{8}$ Yongzhong Xu, ${ }^{1}$ Brian Yanny, ${ }^{2}$ Naoki Yasuda, ${ }^{21}$ Ching-Wa Yip, $^{11}$ D. R. Yocum, ${ }^{2}$ Donald G. York, ${ }^{8,36}$ Idit Zehavi, ${ }^{18}$ Stefano Zibetti, ${ }^{64}$ and Daniel B. Zucker ${ }^{23}$ Received 2004 October 11; accepted 2004 November 18

\section{ABSTRACT}

This paper describes the Third Data Release of the Sloan Digital Sky Survey (SDSS). This release, containing data taken up through 2003 June, includes imaging data in five bands over $5282 \mathrm{deg}^{2}$, photometric and astrometric catalogs of the 141 million objects detected in these imaging data, and spectra of 528,640 objects selected over $4188 \mathrm{deg}^{2}$. The pipelines analyzing both images and spectroscopy are unchanged from those used in our Second Data Release.

Key words: atlases — catalogs — surveys

\footnotetext{
${ }_{1}^{1}$ Theoretical Division, MS B285, Los Alamos National Laboratory, Los Alamos, NM 87545.

2 Fermi National Accelerator Laboratory, P.O. Box 500, Batavia, IL 60510.

3 Department of Astronomy, University of Washington, Box 351580, Seattle, WA 98195.

${ }^{4}$ Apache Point Observatory, P.O. Box 59, Sunspot, NM 88349.

5 Department of Astronomy, MSC 4500, New Mexico State University, P.O. Box 30001, Las Cruces, NM 88003.

${ }^{6}$ Department of Astrophysical Sciences, Princeton University, Princeton, NJ 08544

7 Center for Astrophysical Sciences, Department of Physics and Astronomy, Johns Hopkins University, 3400 North Charles Street, Baltimore, MD 21218.

${ }^{8}$ Department of Astronomy and Astrophysics, University of Chicago, 5640 South Ellis Avenue, Chicago, IL 60637.

${ }_{9}^{9}$ Center for Cosmological Physics, University of Chicago, 5640 South Ellis Avenue, Chicago, IL 60637.

${ }^{10}$ Center for Cosmology and Particle Physics, Department of Physics, New York University, 4 Washington Place, New York, NY 10003.

${ }_{11}^{11}$ Department of Physics and Astronomy, University of Pittsburgh, 3941 O'Hara Street, Pittsburgh, PA 15260.

12 Department of Physics, Carnegie Mellon University, 5000 Forbes Avenue, Pittsburgh, PA 15213.

${ }_{13}$ Magdalena Ridge Observatory, New Mexico Institute of Technology, 801 Leroy Place, Socorro, NM 87801.

14 Department of Astronomy, University of Illinois, 1002 West Green Street, Urbana, IL 61801.

15 Institut d'Estudis Espacials de Catalunya/CSIC, Gran Capitá 2-4, E-08034 Barcelona, Spain.

${ }_{17}$ Department of Physics of Complex Systems, Eötvös Loránd University, Pf. 32, H-1518 Budapest, Hungary.

${ }^{17}$ Institute of Astronomy and Research Center for the Early Universe, School of Science, University of Tokyo, 2-21-1 Osawa, Mitaka, Tokyo 181-0015, Japan.

${ }_{10}^{18}$ Steward Observatory, 933 North Cherry Avenue, Tucson, AZ 85721.

19 Russell-Cotsen Fellow.

${ }^{20}$ Space Telescope Science Institute, 3700 San Martin Drive, Baltimore, MD 21218.

${ }^{21}$ Institute for Cosmic Ray Research, University of Tokyo, 5-1-5 Kashiwa, Kashiwa City, Chiba 277-8582, Japan.

${ }^{22}$ Microsoft Research, 455 Market Street, Suite 1690, San Francisco, CA 94105.

${ }^{23}$ Max-Planck-Institut für Astronomie, Königstuhl 17, D-69117 Heidelberg, Germany.

${ }^{24}$ Lucent Technologies, 2000 Lucent Lane, Naperville, IL 60566.
} 


\section{INTRODUCTION}

The Sloan Digital Sky Survey (SDSS; York et al. 2000) is carrying out an imaging and spectroscopic CCD survey of the

${ }^{25}$ Department of Physics and Astronomy, York University, 4700 Keele Street, Toronto, ON M3J 1P3, Canada.

${ }^{26}$ Department of Mathematical and Physical Sciences, Japan Women's University 2-8-1 Mejirodai, Bunkyo, Tokyo 112-8681, Japan.

27 Department of Astronomy, University of Wisconsin, 475 North Charter Street, Madison, WI 53706.

${ }^{28}$ US Naval Observatory, Flagstaff Station, P.O. Box 1149, Flagstaff, AZ 86002-1149.

${ }^{29}$ Institute for Astronomy and Computational Sciences, Physics Department, Catholic University of America, Washington, DC 20064.

${ }^{30}$ US Naval Observatory, 3540 Massachusetts Avenue NW, Washington, DC 20392.

${ }^{31}$ Code 7215, Remote Sensing Division, Naval Research Laboratory, 4555 Overlook Avenue SW, Washington, DC 20392.

${ }^{32}$ National Astronomical Observatory, 2-21-1 Osawa, Mitaka, Tokyo 1818588, Japan.

33 Astronomical Institute, Tohoku University, Aramaki, Aoba, Sendai 980-8578, Japan.

${ }^{34}$ ISR-4, MS D448, Los Alamos National Laboratory, P.O. Box 1663, Los Alamos, NM 87545.

35 Obserwatorium Astronomiczne na Suhorze, Akademia Pedogogiczna w Krakowie, ulica Podchorążych 2, PL-30-084 Kraców, Poland.

${ }^{36}$ Enrico Fermi Institute, University of Chicago, 5640 South Ellis Avenue, Chicago, IL 60637.

${ }^{37}$ Lawrence Berkeley National Laboratory, One Cyclotron Road, Berkeley, CA $94720-8160$

38 Astronomy Centre, University of Sussex, Falmer, Brighton BN1 9QJ, UK.

39 Department of Physics and Astrophysics, Nagoya University, Chikusa, Nagoya 464-8602, Japan.

${ }_{40}$ SNS-4, MS H820, Los Alamos National Laboratory, P.O. Box 1663, Los Alamos, NM 87545.

${ }^{41}$ Department of Physics, University of Michigan, 500 East University Avenue, Ann Arbor, MI 48109.

${ }^{42}$ Institute for Astronomy, Royal Observatory, University of Edinburgh, Blackford Hill, Edinburgh EH9 3HJ, UK.

${ }^{43}$ Institute for Advanced Study, Olden Lane, Princeton, NJ 08540.

${ }^{44}$ Department of Physics, Applied Physics, and Astronomy, Rensselaer Polytechnic Institute, 110 Eighth Street, Troy, NY 12180.

45 Institute of Cosmology and Gravitation, Mercantile House, Hampshire Terrace, University of Portsmouth, Portsmouth PO1 2EG, UK

${ }^{46}$ CMC Electronics Aurora, 84 North Dugan Road, Sugar Grove, IL 60554.

${ }^{47}$ Department of Astronomy and Research Center for the Early Universe, University of Tokyo, 7-3-1 Hongo, Bunkyo, Tokyo 113-0033, Japan.

48 Joseph Henry Laboratories, Princeton University, Princeton, NJ 08544.

${ }^{49}$ FNRS Institut d'Astronomie et d'Astrophysique, Université Libre de Bruxelles, CP. 226, Boulevard du Triomphe, B-1050 Bruxelles, Belgium.

${ }^{50}$ Department of Physics, Rochester Institute of Technology, 84 Lomb Memorial Drive, Rochester, NY 14623-5603.

${ }^{51}$ UCO/Lick Observatory, University of California, Santa Cruz, CA 95064.

52 Department of Astronomy and Astrophysics, 525 Davey Laboratory, Pennsylvania State University, University Park, PA 16802

53 Center for Astrophysics and Space Astronomy, University of Colorado, Boulder, CO 80309.

54 Japan Participation Group, c/o Institute for Cosmic Ray Research, University of Tokyo, 5-1-5 Kashiwa, Kashiwa City, Chiba 277-8582, Japan.

${ }_{55}$ Department of Physics and Astronomy, University of Wyoming, Laramie, WY 82071

${ }^{56}$ University of Zagreb, Department of Physics, Bijenička cesta 32, 10000 Zagreb, Croatia.

${ }_{57}$ Adler Planetarium and Astronomy Museum, 1300 Lake Shore Drive, Chicago, IL 60605.

58 Institute for Astronomy, 2680 Woodlawn Road, Honolulu, HI 96822.

59 Max-Planck-Institut für extraterrestrische Physik, Giessenbachstrasse 1, D-85741 Garching, Germany.

${ }^{60}$ Department of Physics, University of Pennsylvania, Philadelphia, PA 19104.

${ }^{61}$ Observatories of the Carnegie Institution of Washington, 813 Santa Barbara Street, Pasadena, CA 91101.

62 Department of Physics, Drexel University, 3141 Chestnut Street, Philadelphia, PA 19104.

${ }^{63}$ Department of Astronomy, Ohio State University, 140 West 8th Avenue, Columbus, $\mathrm{OH} 43210$.

${ }^{64}$ Max-Planck-Institut für Astrophysik, Postfach 1, D-85748 Garching, Germany. sky at high Galactic latitudes, using a dedicated wide-field $2.5 \mathrm{~m}$ telescope at Apache Point Observatory in southeast New Mexico. The telescope saw first light in 1998 May, and following an extensive period of commissioning, formal survey operations began in 2000 April. The resulting data have been distributed to the public via Web interfaces accessible from the SDSS public Web site $^{65}$ and have been described in a series of papers:

1. The Early Data Release, including data taken during commissioning (Stoughton et al. 2002, hereafter the EDR paper), consisting of $462 \mathrm{deg}^{2}$ of imaging data and spectra of 54,000 objects.

2. The First Data Release (Abazajian et al. 2003, hereafter the DR1 paper), consisting of imaging data over $2099 \mathrm{deg}^{2}$ and spectra of 186,240 objects.

3. The Second Data Release (Abazajian et al. 2004, hereafter the DR2 paper), consisting of imaging data over $3324 \mathrm{deg}^{2}$ and spectra of 367,360 objects.

The SDSS imaging data are taken on photometric moonless nights (with photometricity determined by an auxiliary telescope; Hogg et al. 2001) of good seeing with a wide-field imaging camera operating in drift-scan mode (Gunn et al. 1998). Six parallel scan lines on the sky, each $13^{\prime}$ wide, are observed by each of the columns of CCDs. Each of the five rows of the CCDs in the camera is fronted by a different filter; thus, each scan line is observed in five filters, denoted (in order of observation) $r, i, u, z$, and $g$ (Fukugita et al. 1996; Gunn et al. 1998; EDR paper). The imaging data are processed by automated software pipelines that measure the properties of all detected objects (Lupton et al. 2001) and perform astrometric (Pier et al. 2003) and photometric calibration, the latter to a set of standard stars observed with the Photometric Telescope (Smith et al. 2002). The resulting object catalogs are used to select targets for spectroscopy, including the main sample of galaxies, magnitudelimited to Petrosian $r=17.77$ (Strauss et al. 2002), a sample of luminous red elliptical galaxies to Petrosian $r=19.5$ (Eisenstein et al. 2001; DR2 paper), and quasar candidates selected by their colors to $i=19.1$ (for $z<3$ candidates) and $i=20.2$ (for higher redshift candidates) (Richards et al. 2002), as well as a host of additional targets, including optical counterparts to ROSAT X-ray sources (Anderson et al. 2003), unusual stars, and calibration observations (EDR paper). All magnitude limits here are corrected for Galactic extinction following Schlegel et al. (1998). The list of spectroscopic targets is distributed among a series of spectroscopic tiles of $3^{\circ}$ diameter (Blanton et al. 2003) to maximize observing efficiency. Each tile then forms the design for a spectroscopic plate: holes are drilled in aluminum plates corresponding to the position of each object for which spectra will be measured. At the telescope, optical fibers feeding a pair of double spectrographs are plugged into each plate; the spectroscopic observations, carried out under conditions not pristine enough for imaging, are typically 45 minutes per plate. The spectra are wavelength- and flux-calibrated and run through an automatic pipeline to classify them and determine redshifts (EDR paper). The previous data release papers describe the quality of the data; the basic attributes of the data are given in Table 1.

\section{THE THIRD DATA RELEASE}

The SDSS Third Data Release (DR3) consists of all surveyquality data taken through 2003 June as part of the main SDSS survey. The footprints of the imaging and spectroscopic data are shown in Figure 1. The spectroscopic footprint is smaller: because spectroscopic targets are chosen from the imaging data,

\footnotetext{
65 See http://www.sdss.org.
} 
TABLE 1

Characteristics of the SDSS Third Data Release (DR3)

\begin{tabular}{|c|c|}
\hline Quantity & Value \\
\hline \multicolumn{2}{|l|}{ Imaging } \\
\hline Footprint area & $5282 \mathrm{deg}^{2}$ \\
\hline 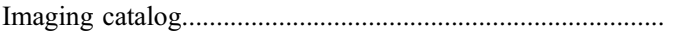 & 141 million unique objects \\
\hline \multicolumn{2}{|l|}{ Magnitude limits: ${ }^{a}$} \\
\hline и ……..................... & 22.0 \\
\hline$g$ & 22.2 \\
\hline$r$ & 22.2 \\
\hline$i$ & 21.3 \\
\hline$z$ & 20.5 \\
\hline Median PSF width & $1 " 4$ in $r$ \\
\hline \multicolumn{2}{|l|}{ RMS photometric calibration errors: } \\
\hline$r$ & $2 \%$ \\
\hline$u-g$ & $3 \%$ \\
\hline$g-r$ & $2 \%$ \\
\hline$r-i$ & $2 \%$ \\
\hline$i-z$ & $3 \%$ \\
\hline 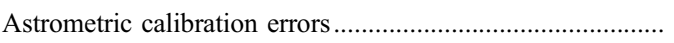 & $<0$."1 $1 \mathrm{rms}$ absolute per coordinate \\
\hline
\end{tabular}

Spectroscopy

\begin{tabular}{|c|c|}
\hline Footprint area ............. & $4188 \mathrm{deg}^{2}$ \\
\hline Wavelength coverage & $3800-9200 \AA$ \\
\hline Resolution $\lambda / \Delta \lambda \ldots \ldots$ & $1800-2100$ \\
\hline Signal-to-noise ratio ................. & $>4$ per $\sim 1 \AA$ pixel at $g=20.2$ \\
\hline Wavelength calibration ............................... & $<5 \mathrm{~km} \mathrm{~s}^{-1}$ \\
\hline Redshift accuracy ${ }^{\mathrm{b}}$ & $30 \mathrm{~km} \mathrm{~s}^{-1} \mathrm{rms}$ for main galaxies \\
\hline Number of spectra & 528640 \\
\hline 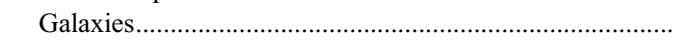 & 374767 \\
\hline 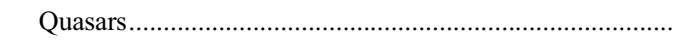 & 51027 \\
\hline Stars & 71174 \\
\hline 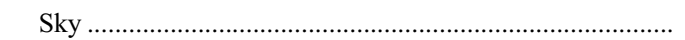 & 26819 \\
\hline Unclassifiable ......................... & 4853 \\
\hline
\end{tabular}

a $95 \%$ completeness for point sources in typical seeing; $50 \%$ completeness numbers are typically 0.4 mag fainter (DR1 paper).

b Approximately $99 \%$ of the classifications and redshifts are reliable.

the spectroscopy always lags the imaging. As with previous data releases, DR3 does not include repeat imaging scans (mostly on the celestial equator in the south Galactic cap; see Fig. 1), repeat observations of spectroscopic plates that have been observed more than once, or imaging or spectroscopic data taken significantly outside the ellipse of the main survey footprint (as described in York et al. 2000). However, six spectroscopic plates, and $34 \mathrm{deg}^{2}$ of imaging data in the north Galactic cap in DR3, lie just outside this ellipse. As in previous data releases, DR3 does include imaging data that overlap between adjacent runs.

The SDSS has taken a considerable amount of imaging data at low Galactic latitudes outside the survey footprint. The subset of these data taken before the summer of 2003 has been made publicly available in a release separate from DR3 and is described by Finkbeiner et al. (2004).

As the survey has progressed, we have steadily improved the software used to process the imaging and spectroscopic data. These changes are described in the DR1 and DR2 papers, and at the public SDSS Web site. Each subsequent release has incorporated all the data included in the previous release, necessitating a reprocessing of those data. For DR3, however, we have made no changes in the processing software, and therefore the DR2 subset of the DR3 data is identical to the data already made public in 2004 March. There is one exception to this statement, namely, that we have updated some incorrect spectral classifica- tions in DR3. We have also added several new auxiliary tables to the SDSS Catalog Archive Server (CAS), which will be useful for those using SDSS data. The Archive Introduction page in the Help menu on the CAS Web site ${ }^{66}$ describes the CAS data model. It has a new link at the top for release-specific notes.

\subsection{Updated Redshifts}

We have visually inspected the spectra of all objects that remained unclassified (spectral class UNKNOWN), and have updated the classification and redshift where appropriate. Many of these objects are of low signal-to-noise ratio or are unusual objects of various types, especially unusual quasars (see Hall et al. 2002). There are 477 objects whose classifications were updated in this effort, including 377 objects included in DR2.

\subsection{New Auxiliary Tables}

We have added a separate database table that includes stellar $B, V, R$, and $I$ Kron-Cousins photometry with an accuracy of $0.02 \mathrm{mag}$ or better from Stetson (2000), as downloaded from the Canadian Astronomy Data Centre photometric standards Web site. ${ }^{67}$ These data, when cross-matched with SDSS, allow SDSS photometry of stars to be compared with an external

\footnotetext{
${ }_{67}^{66}$ See http://skyserver.sdss.org.

${ }^{67}$ See http://cadcwww.dao.nrc.ca/standards.
} 

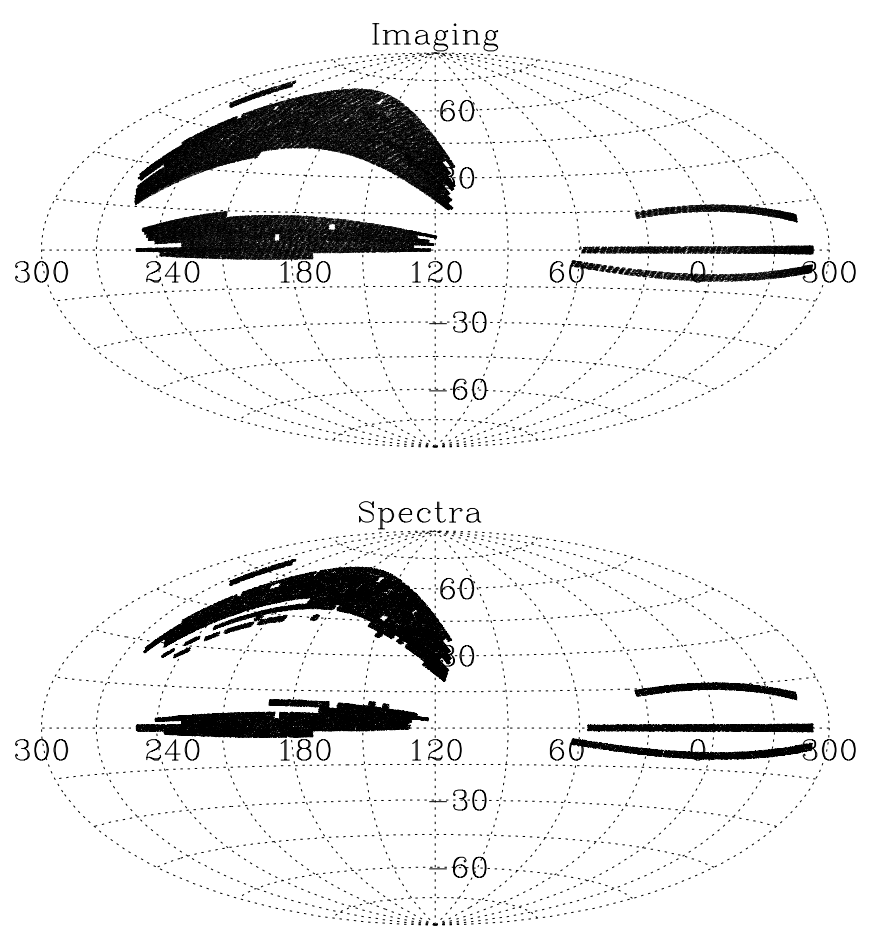

FIG. 1. - Footprint of the SDSS imaging (top) and spectroscopic (bottom) data included in DR3. The former covers $5282 \mathrm{deg}^{2}$, while the latter is $4188 \mathrm{deg}^{2}$. The figure is an Aitoff projection in equatorial coordinates. Note that it wraps at $\alpha=300^{\circ}=20^{\mathrm{h}}$. The data in the south Galactic cap $\left(60^{\circ}>\alpha>300^{\circ}\right)$ consist of three stripes. In the north Galactic cap, the SDSS is working north from the celestial equator and south from a region centered on $\delta \approx+45^{\circ}$.

catalog. After fitting for the conversion between the ugriz SDSS photometric system and the Kron-Cousins photometry used by Stetson, we find the residuals shown in Figure 2; the rms scatter is of order $2.5 \%$ in each band. There is some evidence that this scatter is dominated by errors in the photometric calibration of the SDSS. Further details will be given in J. A. Holtzman et al. (2005, in preparation). We have also included data from the Third Reference Catalog of Galaxies (de Vaucouleurs et al. 1991), again to allow cross-reference to SDSS data. Please see the CAS Archive Introduction page on the SkyServer Web site's Help page for more information about the Stetson and RC3 databases.

\subsection{Imaging Quality Measures on a Field-by-Field Basis}

As part of quality assurance of the SDSS data, each of the $\sim 200,00010^{\prime} \times 13^{\prime}$ fields within DR3 is assigned a quality flag, FieldQAll, which is now made available in a separate table entitled "RunQA" in both the Catalog Archive Server and Data Archive Server. This flag is based on five attributes:

1. The seeing in the $r$ band.

2. The mean offset between the $7^{\prime \prime}$ aperture magnitude, and the point-spread function (PSF) magnitude for bright stars on the frame. The accuracy of the results of the photometric pipeline are critically dependent on a correct model for the PSF, and the aperture minus PSF magnitude is an excellent diagnostic for problems in the PSF determination. This magnitude difference typically is large under conditions in which the seeing is varying rapidly (see the discussion in Ivezić et al. 2004).

3. Systematic offsets of the stellar locus in color space and/or increased width of the stellar locus. As described in Fan (1999), Finlator et al. (2000), Helmi et al. (2003), and many other papers, stars in the SDSS photometric system follow a narrow locus in color-color diagrams. One can define a series of four principal

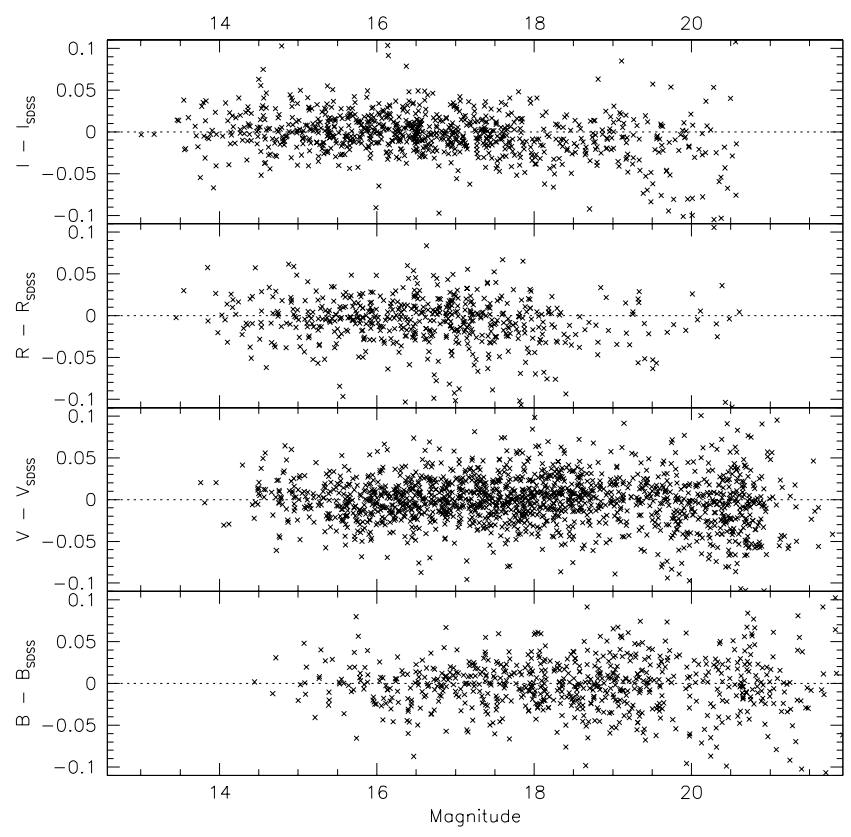

FIG. 2.-Comparison of SDSS and Stetson (2000) photometry of stars. After finding the best-fit conversion from SDSS ugriz photometry to the KronCousins system used by Stetson, the residuals are found to be independent of magnitude (perhaps indicating that they are dominated by photometric calibration and/or the uncertainties in the transformation between the two photometric systems) and have an rms of $\sim 2.5 \%$.

colors from fits to the stellar locus, which are linear transformations of the SDSS magnitudes, which empirically are essentially constant over the sky (after correcting for foreground reddening following Schlegel et al. [1998]). That is, systematic deviations of these principal colors as small as $1 \%$ could indicate problems in the data. These principal colors are defined as:

A. The $s$ color, $s=-0.249 u+0.794 g-0.555 r+0.234$, which is perpendicular to the stellar locus in the $(u-g, g-r)$ diagram.

B. The $w$ color, $w=-0.227 g+0.792 r-0.567 i+0.050$, which is perpendicular to the blue branch of the stellar locus in the $(r-i, g-r)$ diagram.

C. The $x$ color, $x=0.707 g-0.707 r-0.983$, which is perpendicular to the red branch of the stellar locus in the $(r-i$, $g-r$ ) diagram.

D. The $y$ color, $y=-0.270 r+0.800 i-0.534 z+0.059$, which is perpendicular to the stellar locus in the $(r-i, i-z)$ diagram.

These principal colors are measured directly from the stars in running bins four fields wide. Deviations from the global mean principal colors are indications of photometric errors, especially those due to photometric calibration. Scatter around the principal colors within a single bin (i.e., a broad stellar locus) is another indication of poor data. For the present, we use the so-called $s$ color in our overall quality assessment of each field (although statistics on all four colors are available in the RunQA table). In future work, we plan to incorporate information about all four colors in the field quality.

4. Finally, the processing of the data itself can indicate problems. As described in $\S 4.6$ of the EDR paper ( $\S 4.6$ ), a field can be given a so-called operational database quality of BAD, MISSING, or HOLE, often because the data corresponding to the field are particularly poor or the photometric pipeline timed out on the processing (e.g., because of the presence of a naked-eye star in the field). 
Based on these quantities, we assign FieldQAll for each field, as follows:

1. By default, the field in question is assigned FieldQAll = ACCEPTABLE (listed in the RunQA table numerically as 1).

2. If the absolute value of the median PSF-aperture difference is greater than $0.05 \mathrm{mag}$ in any of the five bands; the absolute value of the $s$ color median is larger than 0.05 mag; the $s$ color distribution width is more than 2.5 times larger than its median value for the whole run; the $r$ band seeing is worse than $3^{\prime \prime}$; or the operational database quality is BAD, MISSING, or HOLE, the field in question is downgraded to BAD (listed as 0).

3. If the absolute median PSF-aperture difference is smaller than 0.03 mag in all five bands; the absolute value of the $s$ color median is smaller than $0.03 \mathrm{mag}$; and the $s$ color distribution width is smaller than twice its median value for the whole run, the field in question is upgraded to GOOD (listed as 2).

4. If the median PSF-aperture difference is smaller than 0.02 mag in all five bands; the absolute value of the $s$ color median is smaller than $0.02 \mathrm{mag}$; the $s$ color distribution width is smaller than 1.5 times its median value for the whole run; and the $r$-band seeing is better than $2^{\prime \prime}$, the field in question is upgraded to EXCELLENT (listed as 3 ).

In the DR3, 58\% of fields are EXCELLENT, 26\% are GOOD, $13 \%$ are ACCEPTABLE, and only 3\% are BAD.

Examples of how to use the RunQA table can be found by selecting this table in the CAS Schema Browser on the SkyServer Web site.

\section{LOOKING TO THE FUTURE}

Our next data release after DR3 will consist of data taken through 2004 July; it is anticipated for 2005 July. SDSS survey operations will end at about that time, and a final data release (DR5) is planned for early 2006. As Figure 1 implies, there will still be a substantial gap between the northern and southern pieces of the sky covered in the north Galactic cap (i.e., $110^{\circ}<$ $\alpha<270^{\circ}$ ), and we are actively seeking funds to extend operations beyond the summer of 2005 to fill the gap.

Funding for the creation and distribution of the SDSS archive has been provided by the Alfred P. Sloan Foundation, the Participating Institutions, the National Aeronautics and Space Administration, the National Science Foundation, the U.S. Department of Energy, the Japanese Monbukagakusho, and the Max Planck Society. The SDSS Web site is at http://www.sdss.org.

The SDSS is managed by the Astrophysical Research Consortium (ARC) for the Participating Institutions. The Participating Institutions are The University of Chicago, Fermilab, the Institute for Advanced Study, the Japan Participation Group, The Johns Hopkins University, the Korean Scientist Group, Los Alamos National Laboratory, the Max-Planck-Institute for Astronomy (MPIA), the Max-Planck-Institute for Astrophysics (MPA), New Mexico State University, University of Pittsburgh, Princeton University, the United States Naval Observatory, and the University of Washington.
Abazajian, K., et al. 2003, AJ, 126, 2081 (DR1 paper)

2004, AJ, 128, 502 (DR2 paper)

Anderson, S., et al. 2003, AJ, 126, 2209

Blanton, M. R., Lin, H., Lupton, R. H., Maley, F. M., Young, N., Zehavi, I., \& Loveday, J. 2003, AJ, 125, 2276

de Vaucouleurs, G., de Vaucouleurs, A., Corwin, H. G., Buta, R. J., Paturel, G., \& Fouque, P. 1991, The Third Reference Catalog of Galaxies (Berlin: Springer)

Eisenstein, D. J., et al. 2001, AJ, 122, 2267

Fan, X. 1999, AJ, 117, 2528

Finkbeiner, D., et al. 2004, AJ, 128, 2577

Finlator, K., et al. 2000, AJ, 120, 2615

Fukugita, M., Ichikawa, T., Gunn, J. E., Doi, M., Shimasaku, K., \& Schneider, D. P. 1996, AJ, 111, 1748

Gunn, J. E., et al. 1998, AJ, 116, 3040

Hall, P., et al. 2002, ApJS, 141, 267

Helmi, A., et al. 2003, ApJ, 586, 195

\section{REFERENCES}

Hogg, D. W., Finkbeiner, D. P., Schlegel, D. J., \& Gunn, J. E. 2001, AJ, 122, 2129

Ivezić, Ž., et al. 2004, Astron. Nachr., 325, 583

Lupton, R. H., Gunn, J. E., Ivezić, Ž., Knapp, G. R., Kent, S., \& Yasuda, N. 2001, in ASP Conf. Proc., 238, Astronomical Data Analysis Software and Systems X, ed. F. R. Harnden, Jr., F. A. Primini, \& H. E. Payne (San Francisco: ASP), 269

Pier, J. R., Munn, J. A., Hindsley, R. B., Hennessy, G. S., Kent, S. M., Lupton, R. H., \& Ivezić, Ž. 2003, AJ, 125, 1559

Richards, G. T., et al. 2002, AJ, 123, 2945

Schlegel, D. J., Finkbeiner, D. P., \& Davis, M. 1998, ApJ, 500, 525

Smith, J. A., et al. 2002, AJ, 123, 2121

Stetson, P. B. 2000, PASP, 112, 925

Stoughton, C., et al. 2002, AJ, 123, 485 (EDR paper)

Strauss, M. A., et al. 2002, AJ, 124, 1810

York, D. G., et al. 2000, AJ, 120, 1579 